\title{
Epidemiologische Grundlagen nosokomialer Infektionen
}

\author{
Ralf-Peter Vonberg und Nico Tom Mutters
}

\section{Inhalt}

1 Terminologie

1.1 Epidemiologie ..................................

1.2 Nosokomiale Infektion, Kolonisation, Kontamination,

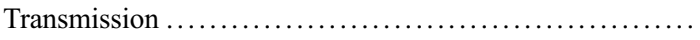

1.3 Prävalenz, Inzidenz, Inzidenzdichte $\ldots \ldots \ldots \ldots \ldots \ldots \ldots$

1.4 Endemie, Epidemie, Pandemie

2 Studientypen

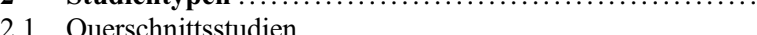

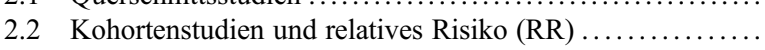

2.3 Fallkontrollstudien und Odds Ratio (OR) ................

2.4 Interventionsstudien (randomisierte, kontrollierte Studien)

2.5 Studien über Studien (systematisches Review und Metaanalysen)

2.6 Zufällige Fehler, systematische Fehler (Bias), Confounder

3 Übertragungswege .

3.1 Einteilung der Erreger....

3.2 Übertragung durch Kontakt (direkt und indirekt) ..........

3.3 Übertragung durch respiratorische Tröpfchen ..............

3.4 Übertragung durch Tröpfchenkerne (Aerosole) ............

3.5 Übertragung durch Umweltmedien (Wasser und Luft)

3.6 Andere Übertragungswege .........................

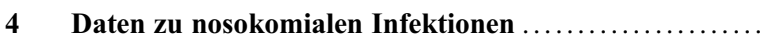

4.1 Im ganzen Krankenhaus .............................

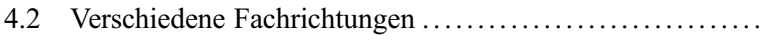

4.3 Intensivstationen

4.4 Erreger in Ausbrüchen (Outbreaks)
$5 \quad$ Folgen nosokomialer Infektionen .................... 11

5.1 Verlängerung der Verweildauer $\ldots \ldots \ldots \ldots \ldots \ldots \ldots \ldots \ldots \ldots \ldots \ldots \ldots$

5.2 Erhöhung der Letalität ............................ 11

Literatur ..................................... 12

Viele Faktoren tragen zu erhöhten nosokomialen Infektionsraten bei. Der Anteil alter Patienten mit chronischen Krankheiten und Immunsupprimierter steigt. Fortschritte in Diagnostik und Therapie resultieren immer häufiger in invasiven Eingriffen. Antibiotikaresistenzen und Folgen nosokomialer Infektionen erfordern daher eine verlässliche Epidemiologie. Konsequenzen nosokomialer Infektionen betreffen einerseits Patienten (Morbidität und Letalität), aber auch das Gesundheitswesen, dem zusätzliche, teilweise vermeidbare finanzielle Belastungen entstehen.

Evidenzbasierte Empfehlungen, bei denen der Einzelfall und örtliche Besonderheiten Berücksichtigung finden, sind hier Voraussetzungen für eine sinnvolle und kosteneffektive Vorgehensweise. Viele Studien haben Häufigkeiten und Folgen nosokomialer Infektionen sowie Präventionsmaßnahmen untersucht. Um die Qualität solcher Studien und die darauf basierenden - oftmals mit Kosten verbundenen - Empfehlungen beurteilen zu können, ist epidemiologisches Wissen unverzichtbar. Dies beinhaltet
R.-P. Vonberg $(\bowtie)$

Medizinische Hochschule Hannover, Institut für Medizinische Mikrobiologie und Krankenhaushygiene, Hannover, Deutschland E-Mail: vonberg.ralf@mh-hannover.de

\section{N.T. Mutters}

Universitätsklinikum Heidelberg, Zentrum für Infektiologie, Sektion Krankenhaus- und Umwelthygiene, Heidelberg, Deutschland

E-Mail: nico.mutters@med.uni-heidelberg.de die Kenntnis der Erreger sowie derer Übertragungswege. Die Epidemiologie nosokomialer Infektionen ist daher die Grundlage ihrer eigenen Verbesserung.

\section{$1 \quad$ Terminologie}

Um die Epidemiologie nosokomialer Infektionen verständlich darstellen zu können, werden zunächst einige wichtige Begriffe erklärt und ggf. durch Beispiele verdeutlicht. 


\subsection{Epidemiologie}

Die Epidemiologie beschäftigt sich mit dem Vorkommen, der Verbreitung und der Verteilung von Krankheiten in der Gesamtbevölkerung oder in Bevölkerungsgruppen (Gordis 2001). Davon ausgehend, dass Erkrankungen nicht zufällig verteilt sind, sondern durch Besonderheiten von Individuen, Gruppen oder dem Umfeld begünstigt werden, dient die Epidemiologie mit drei Methoden der Ermittlung von Ätiologie (Ursache), Ausmaß, Verlauf und Prognose von Krankheiten. Sie ermöglicht auf diese Weise die Bestimmung von Risikofaktoren und den gezielten Einsatz geeigneter Präventionsmaßnahmen.

1. Die deskriptive Epidemiologie (z. B. Surveillance) beschreibt Häufigkeiten von Krankheiten in definierten Populationen in einem zeitlichen oder örtlichen Zusammenhang. Ggf. weisen dabei Erkrankte bestimmte Besonderheiten auf, die sie von nicht Betroffenen unterscheiden.

2. Die analytische Epidemiologie untersucht Hypothesen zur Entstehung von Krankheiten. Sind Häufungen von Erkrankungen bekannt geworden, können mit ihr retrospektiv Risikofaktoren bestimmt werden, die mit einer bestimmten Krankheit vergesellschaftet sind (z. B. Fallkontrollstudien), oder es werden in einem zumeist prospektiven Ansatz Erkrankungen bei Personengruppen verglichen, die sich in einem bestimmten Merkmal unterscheiden (z. B. Kohortenstudien). Diese Untersuchungen geben einen starken Hinweis auf die Ursache oder Verbreitung von Erkrankungen, ohne jedoch einen kausalen Zusammenhang zu beweisen.

3. In der experimentellen Epidemiologie wird die Exposition gegenüber einem Faktor oder einer Maßnahme durch den Untersucher gezielt beeinflusst (z. B. Interventionsstudie) und prospektiv ein möglicher Unterschied der Untersuchungsgruppe gegenüber einer Kontrollgruppe bestimmt. $\mathrm{Ob}$ die dabei gewonnenen Erkenntnisse auch für Personen außerhalb der untersuchten Population Gültigkeit haben, muss jedoch zunächst validiert werden.

\subsection{Nosokomiale Infektion, Kolonisation, Kontamination, Transmission}

Eine Infektion beschreibt das Eindringen und die Vermehrung eines Erregers in einem empfänglichen Wirt. Infektionserreger können exogenen (von außen neu herangetragen) und endogenen (aus der eigenen Keimflora des Wirtes stammend) Ursprungs sein. Meist folgt auf die Infektion eine Reaktion des Wirtes (Immunantwort). Diese kann nach einer Inkubationszeit klinisch in Erscheinung treten (Symptome) oder aber dauerhaft inapparent verlaufen und nur durch gezielte Diagnostik nachweisbar sein. Dabei ist zu beachten, dass auch Personen mit inapparenten Infektionen (z. B. Poliomyelitis) symptomatische oder weitere inapparente Infektionen bei anderen empfänglichen Personen auslösen können.

Definition Bei einer nosokomialen Infektion handelt es sich um eine Infektion, die zum Zeitpunkt der Aufnahme in das Krankenhaus noch nicht existierte und sich auch nicht in ihrer Inkubationsphase befand. Auch Infektionen, die im Krankenhaus erworben wurden, sich aber erst nach Entlassung des Patienten manifestieren, gelten als nosokomiale Infektion. Unerheblich ist dabei, ob diese Infektion auf mangelhafte Hygiene zurückzuführen ist oder ob es sich um ein unvermeidbares Ereignis handelte (Geffers et al. 2002).

Exogene Infektionen können oft durch konsequente Hygienemaßnahmen verhindert werden (z. B. Händedesinfektion). Die Mehrzahl nosokomialer Infektionen sind jedoch endogene Infektionen, d. h., der Patient ist bereits Träger des Erregers (z. B. auf Haut oder Schleimhaut), bevor dieser bei ihm eine Infektion hervorruft. Für endogene Infektionen ist das Reduktionspotenzial durch Hygiene daher deutlich geringer. Aber auch einige Ursachen endogener nosokomialer Infektionen sind durch Hygiene beeinflussbar (z. B. Desinfektion der Haut vor chirurgischen Eingriffen).

Bereits die Surveillance nosokomialer Infektionen und Personalschulung senken Infektionsraten deutlich (Kap. - Surveillance nosokomialer Infektionen). Eine Liste nosokomialer Infektionen mit den Definitionskriterien der CDC zu ihrer Diagnose findet sich im Kap. $>$ Surveillance nosokomialer Infektionen (s. auch Rüden et al. 2003).

Nicht jeder Kontakt mit einem Erreger führt zwangsläufig auch zu einer Infektion. Oft kommt es nur zu einer transienten oder chronischen Kolonisation (Besiedelung, Trägerstatus) des Patienten. Später kann eine Kolonisation in eine Infektion übergehen. Außerdem stellt sie eine Infektionsquelle für andere Patienten dar (Kap. Medizinprodukte: Sichere und umweltschonende Aufbereitung).

Eine Kontamination (Verunreinigung) beschreibt das zeitweilige Vorkommen von Erregern auf Menschen oder Gegenständen. Kontaminationen tragen entscheidend zur Verbreitung von Erregern im Krankenhaus bei (z. B. kontaminierte Hände oder Handschuhe des Personals).

Kommt es zur Erregerübertragung von Patienten auf andere Patienten, hat eine Transmission stattgefunden (vgl. Tab. 2). Molekularbiologische Methoden (Typisierung) können deren gemeinsame Herkunft (Klonalität) nachweisen. Oft sind „Spender“ und „Empfänger“ nachträglich jedoch nicht ermittelbar. Um das beantworten zu können, müssen weitere Aspekte (Aufnahme- bzw. Entlassungsdaten und Screeninguntersuchungen bei stationärer Aufnahme) berücksichtigt werden. Transmissionen können auch zwischen Patienten 
erfolgen, deren Aufenthalt im Krankenhaus gar nicht überlappt, indem der Erreger die Zwischenzeit als Kontamination (z. B. auf einem Stethoskop) überdauert hat. Transmissionen können von Kolonisationen, Kontaminationen oder Infektionen ausgehen.

\subsection{Prävalenz, Inzidenz, Inzidenzdichte}

Die Prävalenz beschreibt das Vorkommen einer Erkrankung innerhalb einer Bevölkerung zu einem bestimmten Zeitpunkt, wird mit Querschnittsuntersuchungen erfasst (Abschn. 2.1) und gibt den Anteil Erkrankter an der Gesamtzahl der Personen an. Sie berücksichtigt nicht den Erkrankungsbeginn, steigt aber mit der Erkrankungsdauer an.

Mit der Inzidenz wird die Anzahl neuer Erkrankungsfälle in einem bestimmten Zeitraum oder pro Anzahl stationärer Aufnahmen beschrieben (Longitudinalstudie, Abschn. 2.2). Sie wird auf die Anzahl aller Personen, die in diesem Zeitraum an dieser Erkrankung hätten erkranken können, bezogen und ist daher eine Maßzahl für das Erkrankungsrisiko. Die Anzahl der Erkrankten zu Beginn sowie die Erkrankungsdauer sind dabei unerheblich. Durch die Erfassung der beobachteten Gefährdungstage für eine nosokomiale Infektion (z. B. Patiententage im Krankenhaus, Tage mit intravasalem Katheter) tragen Patienten mit einer kurzen Liegedauer weniger zur Bezugsgröße im Nenner bei als „Langlieger“ mit längerer Exposition und höherem Erkrankungsrisiko. So ergibt sich die Inzidenzdicte.

\section{Wichtig \\ Prävalenz: Anzahl der Patienten mit nosokomialer Infektion zu einem bestimmten Zeitpunkt bezogen auf die Anzahl untersuchter Patienten. \\ Inzidenz: Anzahl neu aufgetretener nosokomialer In- fektionen in einem definiertem Zeitraum oder Neuerkran- kungen pro Anzahl an Aufnahmen.}

\subsection{Endemie, Epidemie, Pandemie}

Ein endemisches Niveau wird durch fortlaufende Überwachung (Surveillance) bestimmt und beschreibt die durchschnittliche Häufigkeit einer bestimmten Infektion. Es ist von regionalen Gegebenheiten (z. B. Malariarisiko je nach Reiseland) oder auch von Merkmalen einer definierten Population (z. B. HIV-Infektion bei Drogenabhängigen) abhängig.

Treten örtlich und zeitlich begrenzt unerwartet häufig spezifische Infektionen auf, spricht man von einer Epidemie (Ausbruch). Beeinflussende Faktoren sind die Art des Erregers, sein Übertragungsweg, die Zusammensetzung und Häu- figkeit von Kontakten exponierter Personen sowie Ort und Zeit des Geschehens. Oft gibt es eine gemeinsame Quelle (z. B. Legionelleninfektionen aus einem kontaminierten Trinkwassernetz). Der Anteil nosokomialer Infektionen, die in Ausbrüchen auftreten, beträgt 2-10 \% (Haley et al. 1985b; Wenzel et al. 1983).

Abzugrenzen von der Epidemie ist die Pandemie. Hier fehlt die örtliche Begrenzung. Mitunter treten Pandemien weltweit auf (z. B. Infektionen mit dem Influenzavirus). Im Zusammenhang mit nosokomialen Infektionen zeigen z. B. einige klonale MRSA-Linien (Chung et al. 2004) sowie die Übertragungen von SARS-Coronaviren (Varia et al. 2003) pandemischen Charakter.

\section{Studientypen}

Die Epidemiologie bietet wichtige Untersuchungsmethoden zur Risikofaktoranalyse für bestimmte Erkrankungen oder zur Prüfung von Hypothesen der Infektionsentstehung. Je nach Fragestellung ist das Studiendesign zu wählen. Die Datenerfassung kann punktuell oder kontinuierlich erfolgen. Die Studie kann rein beschreibend oder gezielt verändernd (Interventionsstudie) sein. Sollen die Auswirkungen von Veränderungen (z. B. des Umfeldes) auf zukünftige Situationen untersucht werden, so kann ein prospektiver Versuchsansatz gewählt werden. Für abgeschlossene Ereignisse bleibt nur eine retrospektive Beurteilung (Gastmeier und Rüden 2001; Gastmeier und Kramer 2004; Wenzel und Nettleman 1999).

\subsection{Querschnittsstudien}

Querschnittsstudien sind Momentaufnahmen zur Ermittlung der Prävalenz nosokomialer Infektionen. Am Tag der Untersuchung bereits kurierte Infektionen sowie zukünftige Infektionen werden nicht erfasst. Die Erfassungswahrscheinlichkeit einer Infektion ist hier also stark abhängig von der Infektionsdauer. Erst eine wiederholte Durchführung dieser Studie kann durch Veränderungen in der Prävalenz Rückschlüsse auf Trends ermöglichen.

Vorteile von Prävalenzuntersuchungen sind ihr relativ geringer Zeit- und Kostenaufwand. Sie werden daher gerne als orientierende Untersuchung zur Infektionshäufigkeit angewendet und steigern die Aufmerksamkeit beim Personal für die Thematik.

Im dargestellten Beispiel (Abb. 1) beschreibt jede Linie die Aufenthaltsdauer eines Patienten mit einer nosokomialen Infektion. An dem markierten Zeitpunkt werden in einer Querschnittsstudie zwei nosokomiale Infektionen erfasst. Die Häufung von Infektionen zuvor bleibt unerkannt. In einer 
Messung kurz danach wären hingegen gar keine Infektionen gefunden worden. Erst eine engmaschige Surveillance bildet das Infektionsgeschehen verlässlich ab. Die Prävalenz beschreibt nur eine aktuelle Situation und keinen Verlauf. Hervorzuheben als große Prävalenzuntersuchung ist der EU-weite Point Prevalence Survey des ECDC (2013).

\subsection{Kohortenstudien und relatives Risiko (RR)}

Kohortenstudien sind Longitudinalstudien und verfolgen eine definierte Gruppe meistens prospektiv über einen längeren Zeitraum (Exposition bekannt, Outcome untersucht). Meist wird eine kontrollierte Kohortenstudie durchgeführt, in der zusätzlich zur Untersuchungsgruppe mit Exposition auch eine Kontrollgruppe ohne Exposition beobachtet wird. Es werden dann in der Untersuchungsgruppe und einer Kontrollgruppe die Anzahl neuer nosokomialer Infektionen (Inzidenz) und aus dem Verhältnis dieser Raten zueinander

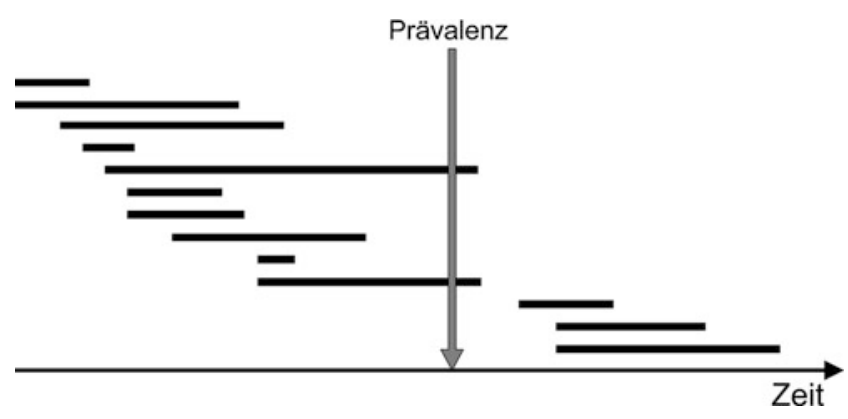

Abb. 1 Prävalenz einer nosokomialen Infektion das relative Risiko (RR) bestimmt. Das RR besagt also, um wie viel häufiger eine Infektion in einer exponierten Gruppe gegenüber einer nicht exponierten Gruppe aufgetreten ist. Tritt in einer exponierten Gruppe eine Infektion häufiger auf, ist die Exposition ein Risikofaktor für den Erwerb der Infektion. Berechnet wird das RR nach der Formel RR = $(a / a+b) /(c / c+d)$. Die Variablen werden der Vierfeldertafel entnommen, die in Tab. 1 dargestellt ist.

Ein $\mathrm{RR}>1$ beschreibt ein erhöhtes, ein $\mathrm{RR}<1$ ein vermindertes Risiko bei Exposition. Werden beim Gruppenvergleich mehrere auffällige Eigenschaften bemerkt, kann mittels multivariater Regressionsanalyse ggf. die Assoziation eines Gruppenunterschiedes mit bzw. die Abhängigkeit von einem anderen Unterschied gefunden werden.

Im Beispiel wird eine Stichprobe von 61 Patienten aus 2 Stationen prospektiv untersucht (Abb. 2). Eine Gruppe von 27 Personen wird auf der Station A versorgt; 4 dieser Patienten entwickeln eine Infektion. Eine Gruppe von 34 Personen wird auf der Station B behandelt; hier entwickeln 22 Personen eine Infektion. Das RR, eine Infektion zu erleiden, beträgt bei einer Behandlung auf Station A in dieser Stichprobe daher $(4 / 27) /(22 / 34)=0,23$, oder anders gesagt: Patienten, die auf der Station B aufgenommen sind, haben ein $(22 / 34) /(4 / 27)=4,37$-mal größeres RR, infiziert zu werden.

Eventuelle Inhomogenitäten zwischen den Gruppen lassen sich durch Stratifizierung, Ausschluss, Matching oder logistische Regressionsanalyse ausgleichen. Die Crossover-Studie, bei der nach der halben Studiendauer Untersuchungs- und Kontrollgruppe die Rollen tauschen und daher gleichermaßen zu beiden Gruppen beitragen, unterstützt den Kausalitätsanspruch der untersuchten Intervention.

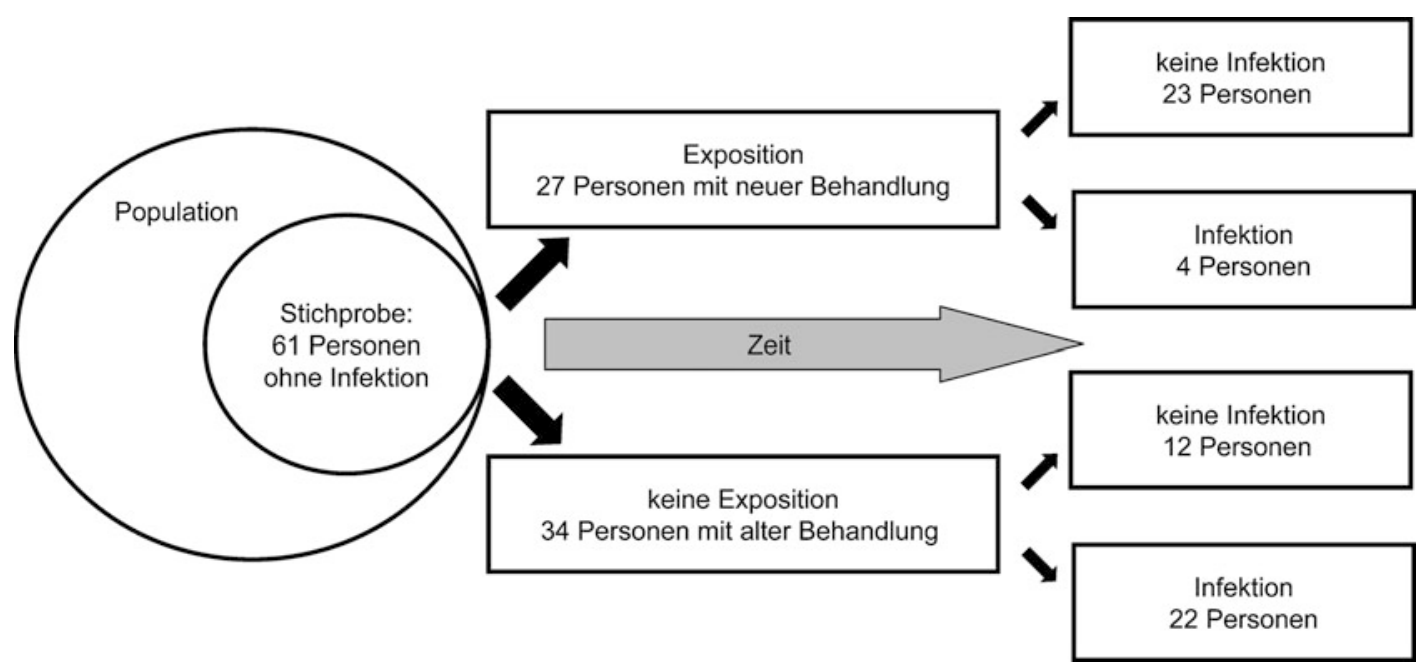

Abb. 2 Beispiel einer Kohortenstudie 
Abb. 3 Fallkontrollstudien

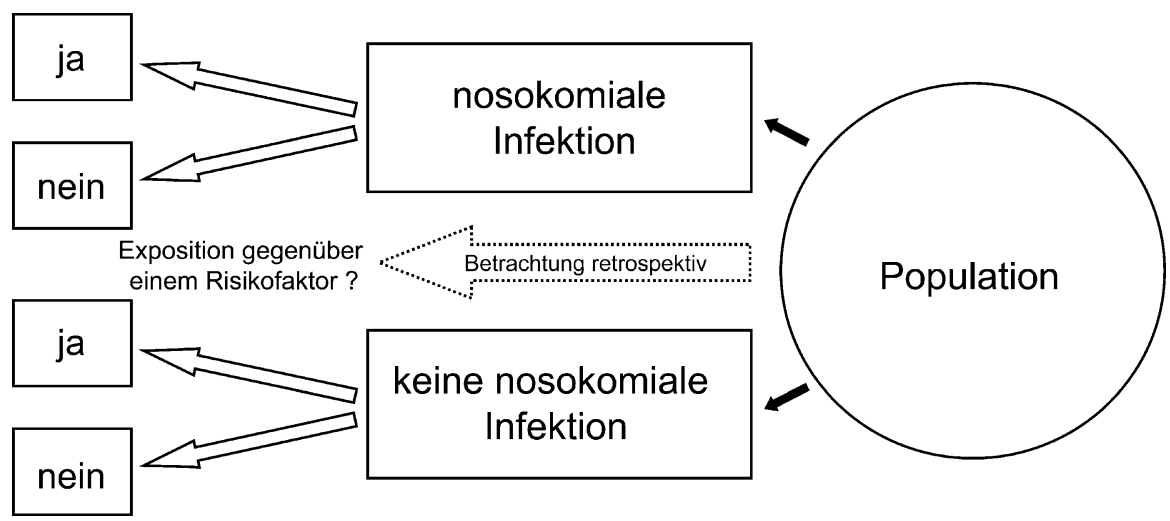

Tab. 1 Vierfeldertafel zur Berechnung des relativen Risikos und der Odds-Ratio

\begin{tabular}{l|l|l}
\hline & Infektion & Keine Infektion \\
\hline Exposition & a & b \\
\hline Keine Exposition & c & d \\
\hline
\end{tabular}

\subsection{Fallkontrollstudien und Odds Ratio (OR)}

Fallkontrollstudien haben einen retrospektiven Ansatz. Es ist zur Infektion gekommen (Outcome bekannt), aber nicht alle Personen sind betroffen. Rückblickend sollen nun Risikofaktoren ermittelt werden, die betroffene Personen kennzeichnen (Exposition untersucht). Diese Studienform ist geeignet zur Untersuchung eines Ausbruchs (Abb. 3). Jedem Betroffenen (Fälle) werden ein oder mehrere Nichtbetroffene gegenübergestellt (Kontrollen). Kontrollen sollten dabei Individuen sein, die demselben Risiko ausgesetzt waren, zu Fällen zu werden - es aber nicht wurden.

Eine besondere Form ist die angepasste Kontrolle (matched case control design), bei der die Kontrollen den Fällen weitestgehend gleichen, abgesehen von den zu untersuchenden Merkmalen. So können z. B. für die Merkmale Alter, Geschlecht oder Schweregrad der Erkrankung passende Kontrollen definiert werden; die Betreuung durch Pflegekräfte hingegen ist nicht abgeglichen, da in dieser Variablen ein Risikofaktor vermutet wird.

Berechnet wird in Fallkontrollstudien die Odds Ratio (OR, Quotenverhältnis) als Schätzwert eines relativen Risikos bei Benutzung der Vierfeldertafel (Tab. 1) nach der Formel OR $=(\mathrm{ad}) /(\mathrm{bc})$. Eine $\mathrm{OR}=1$ spricht dafür, dass kein Zusammenhang zwischen Variable und Infektion besteht. Bei OR $>1$ war das Risiko einer Infektion erhöht, bei OR $<1$ entsprechend erniedrigt.

Retrospektive Ansätze haben den grundsätzlichen Nachteil, auf Fremdinformation aus der Erinnerung und gute Dokumentation in Patientenakten angewiesen $\mathrm{zu}$ sein. Oftmals sind diese Quellen leider lückenhaft (Bias, Abschn. 2.6). Die größte Schwierigkeit bei Fallkontrollstudien ist daher oftmals die korrekte Auswahl der Kontrollen.

\subsection{Interventionsstudien (randomisierte, kontrollierte Studien)}

Evidenz für oder gegen spezifische Maßnahmen leitet sich aus der Qualität der Studien ab, die diese Maßnahmen getestet haben. Je höher die Qualität, desto verlässlicher die ableitbaren Erkenntnisse. Den höchsten Evidenzgrad erzielen randomisierte kontrollierte Interventionsstudien $(\mathrm{RCT}=$ randomized controlled trials).

Das Ziel der Studie muss vor Studienbeginn klar definiert sein. Mit dem $\boldsymbol{\alpha}$-Fehler (meist $5 \%$ ) wird festgelegt, mit welcher Sicherheit ein vermeintlich nachgewiesener Unterschied tatsächlich existiert (entsprechend $95 \%$ ). Eine Stichprobenumfangsberechnung sollte vorab durchgeführt werden, um die erforderliche Anzahl an Patienten für ein signifikantes Ergebnis abschätzen zu können (meist Power der Studie $=80 \%$ bzw. $\boldsymbol{\beta}$-Fehler $=20 \%$ ). Die erforderliche Probandenzahl ist dabei abhängig von dem zu erwarteten nachweisbaren Unterschied: je deutlicher der Unterschied, umso weniger Probanden werden benötigt. So soll vermieden werden, dass in der Studie ein Unterschied übersehen wird, der bei einer größeren Teilnehmerzahl entdeckt worden wäre. Eine unnötig große Teilnehmerzahl hingegen verursacht nur vermeidbaren logistischen und finanziellen Aufwand. Für die Berechnung des erforderlichen Stichprobenumfangs empfiehlt sich geeignete Software oder die Beratung durch einen Experten für medizinische Statistik.

Um den Vorteil neuer Präventionsmaßnahmen gegenüber herkömmlicher Therapien zu überprüfen, werden Patienten zufällig in 2 Gruppen eingeteilt (randomisiert). Es sollen so Patientengruppen gebildet werden, die sich in keinem wesentlichen Merkmal voneinander unterscheiden, außer in der ihnen zu Teil werdenden Therapieform.

Ist den Patienten bekannt, sich in der Studiengruppe zu befinden, kann dies bereits zu einem geänderten Krankheitsempfinden führen (Placeboeffekt). Auch der behandelnde Arzt mit Kenntnis von der Einteilung der Studienpatienten wird dadurch bei der Beurteilung des klinischen Verlaufs beeinflusst. Aus diesem Grund empfiehlt sich, sofern mög- 
lich, die Blindung der Patienten und der behandelnden Ärzte (Doppelblindung) durch eine externe Stelle. Es gibt allerdings auch Studien, bei denen eine Blindung praktisch nicht möglich ist (z. B. Oberkörperhochlagerung zur Prävention der nosokomialen Pneumonie). Prospektiv werden dann die Studien- und Kontrollgruppe beobachtet und beurteilt. Anschließend muss geklärt werden, ob die Ergebnisse grundsätzlich in sich schlüssig sind und korrekt ermittelt wurden (interne Validität) und ob sie auch auf andere Patientengruppen, die evtl. andere gruppenspezifische Merkmale aufweisen, übertragbar sind (externe Validität).

\section{Tipp}

In vielen Studien wird ein $\alpha$-Fehler von $5 \%$ und ein $\beta$-Fehler von $20 \%$ festgelegt, d. h. in $5 \%$ der Studien wird ein Unterschied signifikant nachgewiesen, der gar nicht vorhanden ist, und in $20 \%$ der Studien bleibt ein Unterschied unbemerkt. Dies entspricht bei einem Feuermelder Fehlalarm in $5 \%$ seiner Warnungen und dem Nichterkennen eines jeden 5. Brandes (Beck-Bornholdt und Dubben 2001).

Für gute Studienqualität bei RCTs sprechen:

- eindeutige Zielsetzung,

- Randomisierung,

- Doppelblindung,

- Validierung.

\subsection{Studien über Studien (systematisches Review und Metaanalysen)}

Zur Beantwortung einer epidemiologischen Fragestellung sollte eine Literaturrecherche systematisch und nachvollziehbar erfolgen. Ziel dabei ist, alle relevanten Publikationen von Studien und Fallberichten zu erfassen und nach dem Grad ihrer jeweiligen Qualität geordnet zu bewerten. So entsteht ein systematisches Review (z. B. Dettenkofer et al. 2004). Als Quellen können z. B. Datenbanken im Internet benutzt werden. Zudem sollten die Literaturverzeichnisse der auf diese Weise ermittelten Studien auf weitere wichtige Quellen hin gesichtet werden. Dabei muss bedacht werden, dass Studien mit signifikanten Ergebnissen wahrscheinlicher veröffentlicht werden als solche ohne (Publikationsbias, Abschn. 2.6), was die tatsächliche Datenlage verzerren kann.

Die Aussagekraft einer Studie ist abhängig von der Anzahl der untersuchten Teilnehmer. Mehrere einzelne Studien mit kleinen Gruppen bleiben einzeln betrachtet vielleicht ohne signifikantes Ergebnis, in der Summe ist jedoch ein Einfluss der Intervention nachweisbar. Die Metaanalyse (eine Erweiterung von systematischen Reviews) ist ein Verfahren, um verschiedene Studien zusammenzufassen.

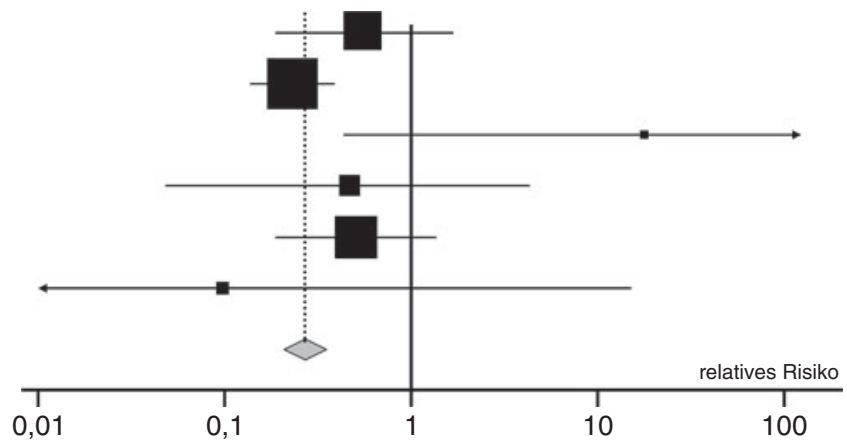

Abb. 4 Forest-Plot einer Metaanalyse

Eine häufig gewählte Darstellung in Metaanalysen ist der Forest-Plot (Abb. 4).

In diesem Beispiel sind 6 Studien in die Metaanalyse eingegangen, in denen vergleichbare Interventionen, Beobachtungen und Messungen erfolgt sind. Jede Studie wird durch ein Quadrat auf einer Linie dargestellt. Quadrate links der markierten „1“ auf der Achse des relativen Risikos sprechen für einen Vorteil, Quadrate rechts der „1“ für einen Nachteil der getesteten Intervention. Die Größe des Quadrats repräsentiert die jeweilige Teilnehmerzahl, die Länge der Linie zeigt das Konfidenzintervall. Dieses beschreibt die Zuverlässigkeit, mit der das ermittelte Ergebnis (Quadrat) korrekt beziffert ist. Je größer das Quadrat, umso kürze ist meistens die dazugehörige Linie (Studien mit vielen Teilnehmer erzielen verlässlichere Ergebnisse). Kreuzt ein Konfidenzintervall die „1“, so kann die Studie kein signifikantes Ergebnis vorweisen, da das vergleichbare Risiko zur Kontrollgruppe möglicherweise den Faktor „1“ beträgt (also gar kein Unterschied vorliegt). Das Ergebnis der Metaanalyse ist der Rhombus. In ihm sind alle Teilnehmer aller Studien verrechnet. Die Länge des Rhombus beschreibt sein Konfidenzintervall. Einzelstudien mit großen Teilnehmerzahlen haben bei der Ermittlung der gepoolten Daten ein entsprechend höheres Gewicht als kleine Studien.

Im gewählten Beispiel zeigt nur die zweite Studie einen signifikanten Vorteil, alle andere Konfidenzintervalle kreuzen die „1“. Die Metaanalyse zeigt insgesamt ebenfalls einen signifikanten Vorteil, obwohl die kleine Einzelstudie 3 (für sich betrachtet) tendenziell einen Nachteil der Intervention zeigte.

\subsection{Zufällige Fehler, systematische Fehler (Bias), Confounder}

Das Auftreten zufälliger Fehler in epidemiologischen Studien kann nie gänzlich ausgeschlossen werden. Je größer die untersuchte Stichprobe, desto geringer ist jedoch der Anteil einzelner „Ausreißer“.

Im Gegensatz dazu resultiert der systematische Fehler (Bias) aus einer Tendenz, bestimmte Ergebnisse regelmäßig 
zu bevorzugen. Dazu einige Beispiele: Ein Publikationsbias liegt vor, wenn Studien, die den Effekt einer Exposition oder die Wirksamkeit einer Intervention beschreiben, häufiger veröffentlicht werden als Studien ohne einen solchen Nachweis. Bei der Erstellung von Metaanalysen muss das bedacht werden, da die Einbeziehung nicht veröffentlichter Studien mitunter nennenswert zum Gesamtergebnis beitragen kann (Kiroff 2001). Der Publikationsbias tritt leicht bei systematischen Reviews und Metaanalysen auf. Retrospektive Studien bergen die Gefahr des Recallbias. Schwer Erkrankte erinnern sich bei Befragungen oft besser an die Exposition gegenüber einem Risikofaktor als weniger stark Erkrankte (Coughlin 1990).

Confounder hingegen sind Kovariablen, die sowohl mit der Intervention (oder Exposition) als auch dem Ergebnis assoziiert sind und daher das Ergebnis (Outcome) beeinflussen. Ein Beispiel für einen Confounder ist das vermehrte Auftreten von Karzinomen bei Kaffeetrinkern - nicht weil Kaffee kausal Krebs verursacht, sondern weil unter Kaffeetrinkern deutlich häufiger Raucher zu finden sind als unter Menschen, die keinen Kaffee trinken (Vineis 1999).

\section{3 Übertragungswege}

Zur Übertragung von Infektionserregern ist stets eine Erregerquelle (z. B. besiedelte oder infizierte Patienten, Besucher oder Mitarbeiter sowie Kontaminationen oder Keimreservoire aus der Umwelt) erforderlich (Abschn. 1.2). Für effektive Präventionsmaßnahmen muss der Übertragungsweg des spezifischen Erregers bekannt sein. Beispiele für die Übertragung von Erregern auf verschiedenen Transmissionswegen zeigt die Tab. 2. Oft führen schon begrenzte, aber konsequent angewandte Maßnahmen, z. B. Händehygiene oder Isolationsmaßnahmen, zur Senkung der Infektionsrate oder zur Beendigung des Ausbruchs. Der dritte bedeutsame Faktor ist der empfängliche Wirt. Gerade hier hat sich in den letzten Jahren viel verändert. So ist z. B. der Anteil multimorbider Patienten deutlich gestiegen, und es gibt zunehmend Patienten mit immunsuppressiver Therapie nach Organtransplantationen.

\subsection{Einteilung der Erreger}

Viren sind Partikel aus Nukleinsäuren (DNA oder RNA) und Proteinen (umgebendes Kapsid) ohne eigenen Stoffwechsel. Sie dringen in Wirtszellen ein und reproduzieren sich unter Nutzung derer Stoffwechselapparate und ggf. unter Schädigung dieser Zellen. Eigenständig vermehren sich Viren nicht. Für die Entwicklung nosokomialer Infektionen ist bedeutsam, ob ein Virus um sein Kapsid herum zusätzlich behüllt oder nicht behüllt (nackt) ist. Während behüllte Viren (z. B. das Influenzavirus) mit den üblichen alkoholischen Desinfektionsmitteln $(70 \mathrm{Vol} \%)$ innerhalb von $30 \mathrm{~s}$ inaktiviert werden können, müssen bei unbehüllten Viren (z. B. Noroviren) entweder längere Einwirkzeiten eingehalten oder auch höhere Konzentrationen verwendet werden (Kap. D Umweltschonende Krankenhausreinigung und Flächendesinfektion).

Bakterien sind einzellige Erreger ohne Zellkern (Prokaryonten), die über einen eigenen Stoffwechsel verfügen. Ihre Erbinformation in Form von DNA ist auf einem Chromosom kodiert und wird bei der Vermehrung stets an die Nachkommen (vertikal) weitergegeben. Weitere Informationen können extrachromosomal auf Plasmiden vorliegen. Diese Plasmide können durch Konjugation auch an bereits existente Bakterien übertragen werden (horizontal), mitunter sogar auf

Tab. 2 Transmissionswege von Infektionserregern

\begin{tabular}{|c|c|c|c|}
\hline \multicolumn{3}{|c|}{ Transmissionsweg } & \multirow{2}{*}{$\begin{array}{l}\text { Erreger (Beispiel) } \\
\text { S. aureus } \\
\text { E. coli }\end{array}$} \\
\hline \multirow[t]{7}{*}{ Horizontal } & \multirow[t]{2}{*}{$\begin{array}{l}\text { Direkter Kontakt von Mensch zu } \\
\text { Mensch }\end{array}$} & Hände & \\
\hline & & Geschlechtsverkehr & $\begin{array}{l}\text { N. gonorrhoeae } \\
\text { T. pallidum }\end{array}$ \\
\hline & \multirow[t]{3}{*}{ Indirekter Kontakt } & $\begin{array}{l}\text { Kontaminierte Gegenstände oder } \\
\text { Flächen }\end{array}$ & $\begin{array}{l}\text { Enterokokken } \\
\text { C. difficile (z. B. durch unzureichend aufbereitete } \\
\text { Medizinprodukte) } \\
\text { Hepatitis-B-Virus (z. B. durch mit Blut verunreinigte } \\
\text { Produkte) }\end{array}$ \\
\hline & & Umweltmedien & $\begin{array}{l}\text { L. pneumophila (Wasser) } \\
\text { A. fumigatus (Luft) }\end{array}$ \\
\hline & & Vektoren & $\begin{array}{l}\text { B. burgdorferi (Schildzecken) } \\
\text { P. falciparum (Stechmücke) }\end{array}$ \\
\hline & \multicolumn{2}{|l|}{ Respiratorische Tröpfchen } & $\begin{array}{l}\text { N. meningitidis } \\
\text { Respiratorische Viren }\end{array}$ \\
\hline & \multicolumn{2}{|l|}{ Tröpfchenkerne (Aerosol) } & $\begin{array}{l}\text { M. tuberculosis } \\
\text { Varizella-zoster-Virus }\end{array}$ \\
\hline Vertikal & \multicolumn{2}{|l|}{ Intrauterin von Mutter zu Kind } & T. gondii (bei Erstinfektion in der Schwangerschaft) \\
\hline
\end{tabular}


andere Arten oder Gattungen. Diese Möglichkeit trägt entscheidend zur leichten und schnellen Verbreitung von Pathogenitätsmechanismen bei, wie z. B. einer Resistenz gegenüber $\beta$-Laktam-Antibiotika bei ESBL-bildenden Enterobakterien.

Die meisten nosokomialen Infektionen werden durch fakultativ pathogene Bakterien verursacht, d. h., sie werden häufig erst bei einer Abwehrschwäche des Wirtes oder bei einer Durchbrechung natürlicher Barrieren (Operation, ZVK Anlage etc.) relevant. Bedeutsam für die Übertragungswahrscheinlichkeit und die Entstehung nosokomialer Infektionen ist die Infektionsdosis (Anzahl erforderlicher Erreger für eine Infektion) sowie die Fähigkeit, in der Umwelt infektiös bleiben zu können (Tenazität). Sporen als hypometabolische Dauerform einiger Bakterienarten (z. B. Clostridien) sind sogar extrem stabil gegenüber physikalischen oder chemischen Noxen, können über viele Jahre infektiös bleiben und erfordern auch bei der Auswahl eines geeigneten Reinigungs- und Desinfektionsmittels besondere Beachtung (Kap. - Umweltschonende Krankenhausreinigung und Flächendesinfektion).

Im Gegensatz zu Bakterien sind Pilze Eukaryonten, d. h., sie verfügen über einen Zellkern. Weitere Unterschiede zeigen sich in der Zusammensetzung der Zellwände. So können in Pilzen Polysaccharide (Glukane und Mannane) sowie Chitinpolymere vorhanden sein. In der medizinischen Mikrobiologie werden Sprosspilze (Hefen), Schimmelpilze (Fadenpilze), Dermatophyten und dimorphe Pilze unterschieden. Bei nosokomialen Mykosen treten hauptsächlich Hefen und Schimmelpilze in Erscheinung.

Cave Besonders gefährdet durch Mykosen sind Immunsupprimierte unter längerer antibiotischer Therapie.

\section{2 Übertragung durch Kontakt (direkt und indirekt)}

Die mit Abstand häufigste Übertragung von Erregern findet durch Berührung statt, sei es die direkte Übertragung durch nicht desinfizierte Hände des Personals (häufigste Übertragung von Erregern im Krankenhaus) oder der indirekte Kontakt über kontaminierte Gegenstände.

\section{3 Übertragung durch respiratorische Tröpfchen}

Bei einer Tröpfcheninfektion wird der Erreger beim Sprechen, Niesen oder Husten in großen Tröpfchen $(>5 \mu \mathrm{m})$ freigesetzt. Der Schwerkraft folgend fallen diese Tröpfchen nach kurzer Distanz (max. 2 m) zur Erde oder auf die nächstgelegene horizontale Fläche. Je nach Erreger bleiben sie dort unterschiedlich lange infektiös und können mittels Kontaktübertragung weiterverbreitet werden. Eine direkte Infektion durch Tröpfchen ist nur dann möglich, wenn eine empfängliche Person sich innerhalb des Streuradius einer infizierten Person befindet. Es empfiehlt sich daher, entweder genügend Abstand einzuhalten oder bei der Tätigkeit am Patienten einen chirurgischen Mund-Nasen-Schutz zu verwenden.

\section{4 Übertragung durch Tröpfchenkerne (Aerosole)}

Tröpfchenkerne $(<5 \mu \mathrm{m})$ entstehen bei der Verdunstung von größeren respiratorischen Tröpfchen. Bleiben in diesen Kernen Erreger über längere Zeit infektiös erhalten und werden von exponierten Personen bis in die Alveolen inhaliert, so kann es zu einer aerogen erworbenen Infektion kommen. Im Gegensatz zu Tröpfchen sinken Tröpfchenkerne kaum zu Boden, sondern bleiben wegen ihres geringen Gewichtes in der Schwebe (Aerosol). So können sich die Erreger über größere Distanzen (sowohl örtlich als auch zeitlich) verbreiten. Als Präventionsmaßnahme bietet sich hier u. a. die Verwendung spezieller Atemschutzmasken an. Ein typisches Anwendungsbeispiel ist die Versorgung von Patienten mit einer offenen Lungentuberkulose (Kap. > Tuberkulose und andere durch Luft übertragbare Infektionserkrankungen: Krankenhaushygiene zur Vermeidung und Eindämmung).

\section{5 Übertragung durch Umweltmedien (Wasser und Luft)}

Auch aus Quellen der Umwelt können Erreger zu nosokomialen Infektionen führen. Typische Beispiele sind Legionellosen aus dem Trinkwassernetz oder Aspergillosen durch in der Luft vorhandene Schimmelpilzsporen (z. B. bei Bauarbeiten freigesetzt). Besonders bei immunologischen Hochrisikopatienten (z. B. nach Transplantationen von Knochenmark oder Organen) muss die Exposition gegenüber solchen Erregern verhindert werden (Kap. Immunsuppression: Hygienische Maßnahmen).

\subsection{Andere Übertragungswege}

Auch durch kontaminierte Nahrungsmittel kann es zu Infektionen im Krankenhaus kommen. Diese Infektionen sind aber, wie auch Übertragungen durch tierische Vektoren, im Zusammenhang mit nosokomialen Infektionen eher selten. Es sind jedoch vereinzelt Infektionen dieser Art z. B. mit 
Hepatitis-A-Viren oder Malaria beschrieben worden (Moro et al. 2002; Petrosillo et al. 2002).

\section{Daten zu nosokomialen Infektionen}

Patienten mit Devices, also Fremdkörpern zu diagnostischen oder therapeutischen Zwecken wie z. B. Beatmungstubus, zentraler Venenkatheter (ZVK) oder Harnwegskatheter (HWK), sowie Patienten auf Intensivstationen und in immunologischen Risikobereichen (z. B. Frühgeborene oder hämatologisch-onkologische Patienten) erleiden besonders häufig nosokomiale Infektionen. Das Erregerspektrum und die Infektionsraten zeigen daher deutliche Unterschiede zwischen Intensivpatienten und Patienten auf peripheren Stationen und zudem auch im Vergleich verschiedener Fachrichtungen. Diesem Problem trägt das Krankenhaus-InfektionsSurveillance-System (KISS) Rechnung (Gastmeier et al. 2012). Weiterführende Informationen über KISS finden sich im Kap. - Surveillance nosokomialer Infektionen und auf der Seite des Nationalen Referenzzentrums für die Surveillance von nosokomialen Infektionen (http://www.nrz-hygiene.de).

Schätzungen zufolge sind etwa $15-30 \%$ aller nosokomialen Infektionen grundsätzlich vermeidbar (Grundmann et al. 2005; Haley et al. 1985a; Harbarth et al. 2003).

\subsection{Im ganzen Krankenhaus}

In einer Prävalenzstudie („Nosokomiale Infektionen in Deutschland - Erfassung und Prävention, NIDEP“) von über 41.000 Patienten aus 132 Krankenhäusern betrug die Gesamtprävalenz nosokomialer Infektionen $3,8 \%$, d. h., auf einer Station mit etwa 30 Betten befindet sich - statistisch immer ein Betroffener (Behnke et al. 2013). Ebenfalls konnte gezeigt werden, dass postoperative Wundinfektionen (24,3\%) und Harnwegsinfektionen (23,2 \%) die mit Abstand häufigsten nosokomialen Infektionen sind, gefolgt von tiefen Atemwegsinfektionen (21,7 \%) (Behnke et al. 2013).

Typische bakterielle grampositive Erreger sind S. aureus, Koagulase-negative Staphylokokken und Enterokokken. Unter den gramnegativen Erregern sind zumeist Enterobakterien (E. coli, Klebsiella spp., Enterobacter spp. und Proteus spp.) sowie P. aeruginosa zu finden.

\subsection{Verschiedene Fachrichtungen}

Unterschiedliche Patientenkollektive haben auch ein verschieden großes Risiko für den Erwerb nosokomialer Infektionen. In Tab. 3 finden sich Beispiele der Inzidenzdichte aus der Literatur und aus dem Modul STATIONS-KISS

Tab. 3 Inzidenzdichte nosokomialer Infektionen in verschiedenen Fachabteilungen

\begin{tabular}{|c|c|c|}
\hline Studie & Abteilung & Infektionen/1000 Beatmungstage \\
\hline \multicolumn{3}{|l|}{ Pneumonie } \\
\hline Kübler et al. (2012) & Intensivpatienten medizinisch/chirurgisch & 18,2 \\
\hline Rogers et al. (2014) & Intensivpatienten pädiatrisch & 30,0 \\
\hline Martínez-Reséndez et al. (2014) & Intensivpatienten medizinisch/chirurgisch & $8,9-17,5$ \\
\hline Balasubramanian und Tullu (2014) & Intensivpatienten pädiatrisch & 6,3 \\
\hline Klompas et al. (2014) & Gemischt & 1,4 \\
\hline \multicolumn{3}{|l|}{ Sepsis } \\
\hline Liu et al. (2010) & Gemischt & $0,2-2,6$ \\
\hline Zimlichman et al. (2013) & Gemischt & 1,72 (mittlere ZVK-assoziierte Sepsisrate) \\
\hline Verstraete et al. (2014) & Intensivpatienten (Neonatologie) & 3,1 \\
\hline Mitt et al. (2014) & Intensivpatienten (Pädiatrie) & 12,8 \\
\hline Modul STATIONS-KISS (2011-2015) & Chirurgie & 0,8 (mittlere ZVK-assoziierte Sepsisrate) \\
\hline Modul STATIONS-KISS (2011-2015) & Innere Medizin & 1,6 (mittlere ZVK-assoziierte Sepsisrate) \\
\hline Modul STATIONS-KISS (2011-2015) & Interdisziplinär & 1,9 (mittlere ZVK-assoziierte Sepsisrate) \\
\hline \multicolumn{3}{|l|}{ Harnwegsinfektion } \\
\hline Edwards et al. (2008) & Intensivpatienten medizinisch/chirurgisch & 3,1 \\
\hline Edwards et al. (2008) & Rehabilitationsklinik & 16,8 \\
\hline Titsworth et al. (2012) & Intensivpatienten (Neurologie) & 13,3 \\
\hline Askarian et al. (2013) & Gemischt & 1,8 \\
\hline Huoi et al. (2013) & Hämatologie & 2,9 \\
\hline Martinez-Resendez et al. (2014) & Intensivpatienten medizinisch/chirurgisch & $12,6-20,3$ \\
\hline Modul STATIONS-KISS (2011-2015) & Chirurgie & 4,4 (mittlere HWK-assoziierte Harnwegsinfektionsrate) \\
\hline Modul STATIONS-KISS (2011-2015) & Innere Medizin & 1,4 (mittlere HWK-assoziierte Harnwegsinfektionsrate) \\
\hline Modul STATIONS-KISS (2011-2015) & Neurologie & 5,6 (mittlere HWK-assoziierte Harnwegsinfektionsrate) \\
\hline Modul STATIONS-KISS (2011-2015) & Interdisziplinär & 1,6 (mittlere HWK-assoziierte Harnwegsinfektionsrate) \\
\hline
\end{tabular}

$H W K$, Harnwegskatheter; $Z V K$, zentraler Venenkatheter 
Tab. 4 Häufigste Erreger Device-assoziierter Infektionen auf Intensivstationen (KISS-Datenbank, 2011-2015; http://www.nrz-hygiene.de)

\begin{tabular}{l|l|l|l}
\hline Rang & Beatmungsassoziierte Pneumonie & ZVK-assoziierte Sepsis & HWK-assoziierte Harnwegsinfektion \\
\hline 1 & S. aureus $(18 \%)$ & Koagulase-negative Staphylokokken $(33 \%)$ & E. coli $(36 \%)$ \\
\hline 2 & P. aeruginosa $(16 \%)$ & Enterococcus spp. $(20 \%)$ & Enterococcus spp. $(27 \%)$ \\
\hline 3 & E. coli $(14 \%)$ & S. aureus $(15 \%)$ & P. aeruginosa $(15 \%)$ \\
\hline
\end{tabular}

$H W K$, Harnwegskatheter; $Z V K$, zentraler Venenkatheter

Tab. 5 Median (25\%- und $75 \%$-Quantil) Device-assoziierter nosokomialer Infektionen, stratifiziert nach Art der Intensivstation, pro 1000 Device-Tage (KISS-Datenbank 2011-2015; http://www.nrz-hygiene.de)

\begin{tabular}{l|l|l|l}
\hline & $\begin{array}{l}\text { Beatmungsassoziierte Pneumonie (invasive } \\
\text { Beatmung) }\end{array}$ & $\begin{array}{l}\text { ZVK-assoziierte Sepsis } \\
(2011-2015)\end{array}$ & $\begin{array}{l}\text { HWK-assoziierte Harnwegsinfektion } \\
(2011-2015)\end{array}$ \\
\hline $\begin{array}{l}\text { Interdisziplinär } \\
(<\mathbf{4 0 0} \text { Betten) }\end{array}$ & $2,50(1,02-4,32)$ & $0,51(0,00-1,10)$ & $0,38(0,00-0,81)$ \\
\hline $\begin{array}{l}\text { Interdisziplinär } \\
(\geq \mathbf{4 0 0} \text { Betten) }\end{array}$ & $3,17(1,86-5,01)$ & $0,80(0,31-1,42)$ & $0,76(0,22-1,33)$ \\
\hline Internistisch & $1,75(0,79-3,07)$ & $0,69(0,00-1,57)$ & $0,36(0,00-0,92)$ \\
\hline Chirurgisch & $3,61(2,13-5,8)$ & $0,75(0,29-1,25)$ & $0,69(0,28-1,26)$ \\
\hline Neurochirurgisch & $4,32(3,30-7,75)$ & $0,68(0,36-1,38)$ & $1,59(0,72-2,53)$ \\
\hline Gesamt & $2,78(1,31-4,86)$ & $0,68(0,18-1,35)$ & $0,51(0,11-1,14)$ \\
\hline
\end{tabular}

$H W K$, Harnwegskatheter; $Z V K$, zentraler Venenkatheter

(Kap. \ Surveillance nosokomialer Infektionen) für deutsche Krankenhäuser.

Die Häufigkeit postoperativer Wundinfektionen ist bekanntermaßen stark abhängig von der Art des durchgeführten Eingriffes (Astagneau et al. 2001). Während im Modul OP-KISS z. B. arthroskopische Eingriffe am Kniegelenk nur sehr selten $(<2 \%)$ eine nosokomiale Wundinfektion nach sich ziehen, kommt es bei fast $8 \%$ der chirurgischen Interventionen am Kolon zu dieser postoperativen Komplikation.

Pro 1000 Patiententage ereignen sich etwa 1,5 Pneumonien, eine Sepsis und eine Harnwegsinfektion. Betrachtet man die Häufigkeit der Harnwegsinfektionen stratifiziert nach verschiedenen Abteilungen, so fällt auf, dass neurologische Patienten deutlich häufiger Harnwegsinfektionen entwickeln als der Durchschnitt. Auch in den Daten des Moduls STATIONS-KISS ist die mittlere Harnwegsinfektionsrate in der Neurologie fast doppelt so hoch wie das Mittel aller Stationen (5,6 vs. 3,2 pro 1000 Harnwegskathetertage). Bei der Beurteilung von Infektionsraten muss daher das untersuchte Kollektiv ebenfalls berücksichtigt werden (hier z. B. Häufigkeit neurogener Blasenentleerungsstörungen).

\subsection{Intensivstationen}

Bei Intensivpatienten stehen schwere, oft lebensbedrohliche nosokomiale Infektionen im Vordergrund und diese sind sehr oft mit Devices assoziiert (Katheter, maschinelle Beatmung). Mehr als die Hälfte aller nosokomialen Infektionen bei Patienten auf Intensivstationen betreffen die tiefen Atemwege, je ungefähr $20 \%$ entfallen auf Sepsis und Harnwegsinfektionen. Die Verteilung der häufigsten Erreger Device-assoziierter Infektionen auf Intensivstationen ist in Tab. 4 aufgeführt.

Verschiedene Intensivstationsarten weisen verschiedene Infektionsraten auf. Die in Tab. 5 genannten Infektionsraten normieren nosokomiale Infektionen auf Device-Anwendungstage. Bei Stationen, die viele beatmete Patienten betreuen, fällt daher eine einzelne Pneumonie eines beatmeten Patienten weniger ins Gewicht als bei Stationen mit nur sehr wenigen intubierten Patienten.

\subsection{Erreger in Ausbrüchen (Outbreaks)}

Ein nosokomialer Ausbruch liegt vor, wenn mehr Infektionen auftreten, als räumlich und zeitlich zu erwarten sind (Ammon et al. 2001). Sein Verlauf wird dabei vom Übertragungsweg, vom Patientenkollektiv, von saisonalen und von regionalen Gegebenheiten beeinflusst. Zur schnellen Orientierung kann im Internet die Outbreak-Database (http://www.outbreakdatabase.com) mit mehr als 3000 publizierten und nach multiplen miteinander verknüpfbaren Kriterien kategorisierten Ausbrüchen genutzt werden. In Tab. 6 sind die häufigsten Infektionsarten in Abhängigkeit von der Altersgruppe der Patienten dargestellt. Dabei ist zu beachten, dass durch einen Erreger auch mehrere Organsysteme betroffen sein können. Nicht jeder Ausbruch lässt sich aufklären: In jedem dritten Ausbruch kann dessen Quelle nicht ermittelt werden; in jedem vierten Ausbruch wird der Übertragungsweg nicht gefunden. 
Tab. 6 Häufigste Infektionsarten in nosokomialen Ausbrüchen in verschiedenen Altersgruppen (aus 3057 Outbreaks; Mehrfachnennungen möglich; http://www.outbreak-database.com)

\begin{tabular}{l|l|l|l|l}
\hline Rang & Neugeborene $(\mathrm{n}=560)$ & Kinder $(\mathrm{n}=386)$ & Erwachsene $(\mathrm{n}=1826)$ & Senioren $(\mathrm{n}=518)$ \\
\hline 1 & Sepsis $(51 \%)$ & Sepsis $(33 \%)$ & Tiefe Atemwege $(30 \%)$ & Tiefe Atemwege $(35 \%)$ \\
\hline 2 & Tiefe Atemwege $(22 \%)$ & Magen/Darm $(28 \%)$ & Sepsis $(30 \%)$ & Magen/Darm $(27 \%)$ \\
\hline 3 & Magen/Darm $(19 \%)$ & Tiefe Atemwege $(27 \%)$ & Magen/Darm $(21 \%)$ & Sepsis $(25 \%)$ \\
\hline 4 & ZNS $(15 \%)$ & Haut $(10 \%)$ & Postoperative Wundinfektion $(15 \%)$ & Haut $(12 \%)$ \\
\hline 5 & Haut $(15 \%)$ & Postoperative Wundinfektion $(10 \%)$ & Harnwege $(13 \%)$ & HNO/Auge $(11 \%)$ \\
\hline
\end{tabular}

\section{$5 \quad$ Folgen nosokomialer Infektionen}

Nosokomiale Infektionen betreffen sowohl den Patienten als auch die Gesellschaft. Kostenaspekte, z. B. durch zusätzlichen Behandlungsaufwand und volkswirtschaftlichen Schaden durch Fehltage oder Minderung der Erwerbsfähigkeit, werden in Kap. $>$ Basishygienemaßnahmen im Krankenhaus behandelt. Für den Patient sind der verlängerter Aufenthalt im Krankenhaus und sein Letalitätsrisiko von Belang.

\subsection{Verlängerung der Verweildauer}

Vergleicht man die Verweildauer von Patienten mit und ohne nosokomialer Infektion, aber ansonsten gleicher Grunderkrankung, kann man die attributive Verlängerung der Verweildauer ermitteln. Die Vergleichbarkeit publizierter Studien ist jedoch nur bedingt zulässig, da die nosokomiale Infektion nicht immer als zeitabhängige Variable aufgefasst und statistisch entsprechend berücksichtigt wird (Beyersmann et al. 2006). Diese Verlängerung der stationären und ggf. intensivmedizinischen Versorgung ist natürlich auch ein entscheidender Faktor bei der Kosten-Nutzen-Kalkulation bei der Einführung von Maßnahmen zur Prävention nosokomialer Infektionen.

In einer Vielzahl von Studien wurden Daten zur zusätzlichen Verweildauer erhoben. Eine nosokomiale Pneumonie auf einer Intensivstation führte - je nach Setting - zu einem 5-26 Tage längeren Aufenthalt und eine nosokomiale postoperative Wundinfektion zu 7-17 zusätzlichen Tagen im Krankenhaus. Das bedeutet in der Regel auch Einnahme von Pharmaka (z. B. Antibiotika) mit der Gefahr von Nebenwirkungen und Unverträglichkeiten sowie eine zusätzliche Zeit unter dem Risiko, weitere nosokomiale Infektionen zu akquirieren. Auch eine Sepsis verlängert den stationären Aufenthalt um bis zu 10 Tage. Dabei muss man jedoch noch berücksichtigen, dass Patienten, die an einer Sepsis erkranken und schnell versterben, sogar verkürzend auf die durchschnittliche Länge der Aufenthaltsdauer im Krankenhaus Einfluss genommen haben (Abschn. 5.2).
Tab. 7 Erhöhte Letalität durch eine nosokomiale Pneumonie (Intensivpatienten)

\begin{tabular}{l|l}
\hline Studie & Zuschreibbare Letalität (\%) \\
\hline Bekaert et al. (2011) & $4,4-5,9$ \\
\hline Melsen et al. (2013) & 13,0 \\
\hline Sopena et al. (2014) & 27,7 \\
\hline Rogers et al. (2014) & 8,7 \\
\hline Behnia et al. (2014) & 37,0 \\
\hline
\end{tabular}

Tab. 8 Erhöhte Letalität durch eine nosokomiale Sepsis

\begin{tabular}{l|l|l}
\hline Studie & Nosokomiale Infektionsart & $\begin{array}{l}\text { Zuschreibbare } \\
\text { Letalität (\%) }\end{array}$ \\
\hline $\begin{array}{l}\text { Barnett et al. } \\
(2013)\end{array}$ & Sepsis & 6,1 \\
\hline $\begin{array}{l}\text { Blennow } \\
\text { et al. (2013) }\end{array}$ & $\begin{array}{l}\text { Sepsis } \\
\text { (Knochenmarkstransplantation) }\end{array}$ & 3,3 \\
\hline $\begin{array}{l}\text { Olaecha et al. } \\
(2013)\end{array}$ & Sepsis (Intensivpatienten) & 9,4 \\
\hline $\begin{array}{l}\text { Stevens et al. } \\
(2014)\end{array}$ & Sepsis & 19,4 \\
\hline $\begin{array}{l}\text { Kaye et al. } \\
(2014)\end{array}$ & $\begin{array}{l}\text { Sepsis (Patienten }>65 . \\
\text { Lebensjahr) }\end{array}$ & 16,2 \\
\hline
\end{tabular}

\subsection{Erhöhung der Letalität}

Um die einer nosokomialen Infektion zuschreibbare zusätzliche Letalität (Exzessletalität; ,attributable mortality“) zu ermitteln, muss sichergestellt werden, dass Patienten mit nosokomialer Infektion nur ansonsten vergleichbar kranken Patienten (ohne Infektion) gegenübergestellt werden (Feinstein 1985). Die Wahrscheinlichkeit, bereits an der Grundkrankheit zu versterben, sollte in beiden Gruppen gleich groß sein; überzählige Todesfälle wären dann ausschließlich auf die Infektion (z. B. Sepsis) zurückzuführen. Die entsprechenden Daten zeigen die Tab. 7, 8 und 9. Die höchste Letalität durch nosokomiale Infektionen mit etwa $25 \%$ findet sich bei der Sepsis. Umgekehrt bedeutet das: Es müssen nur 4 nosokomiale Sepsisfälle vermieden werden, um einen Todesfall zu verhindern.

Bei der Beurteilung der zuschreibbaren Letalität durch eine nosokomiale Pneumonie auf Intensivstationen zeigt die 
Tab. 9 Erhöhte Letalität durch eine nosokomiale postoperative Wundinfektion

\begin{tabular}{l|l}
\hline Studie & Zuschreibbare Letalität (\%) \\
\hline McGarry et al. (2004) & 16,8 \\
\hline Lee et al. (2006) & 13,0 \\
\hline Geffers et al. (2008) & 2,8 \\
\hline Kaye et al. (2009) & 10,1 \\
\hline
\end{tabular}

Studienlage ein besonders heterogenes Bild. In einigen Studien konnte keine erhöhte Letalität gefunden werden (Rello et al. 2002, 2003; Melsen et al. 2009). Bei anderen Untersuchungen verstarb jeder dritte oder vierte Patient mit nosokomialer Pneumonie (Sopena et al. 2014; Behnia et al. 2014); wenn man sich bei den betrachteten Erregern nur auf Pseudomonaden oder Acinetobacter spp. beschränkte, betrug die Exzessletalität sogar 42,8 \% (Fagon et al. 1993). In einer späteren Studie konnte diese stark erhöhte Letalität bei Pneumonien durch Acinetobacter spp. im Vergleich zu anderen Erregern jedoch nicht bestätigt werden (Garnacho et al. 2003). Insgesamt wird diese Problematik aktuell kontrovers diskutiert (Behnia et al. 2014), und sicherlich müssen in neueren Studien weitere Faktoren wie Komorbiditäten genauer einbezogen werden (Guzman-Herrador et al. 2014). Das Erregerspektrum nimmt also entscheidend Einfluss auf die Prognose der Pneumonie, und es variiert stark je nach Disziplin. Umso wichtiger zur Wahl der kalkulierten Therapie ist daher die Kenntnis über die lokale Epidemiologie inklusive der Resistenzsituation. Einheitliche Diagnosekriterien für nosokomiale Infektionen sind zudem erforderlich, um deren Vergleichbarkeit gewährleisten zu können (Timsit et al. 1996; Lewis 1995; Roberts et al. 1998).

Für nosokomiale Harnwegsinfektionen wurde (selbst bei Intensivpatienten) zumeist in älteren Studien keine (BuenoCavanillas et al. 1994; Daschner et al. 1978; Gross und Van Antwerpen 1983; Laupland et al. 2002) oder nur eine mäßig (5\%) erhöhte attributive Letalität beschrieben (Rosenthal et al. 2003). Ob die Resistenzentwicklung bei Erregern von Harnwegsinfektionen hier zukünftig zu Veränderungen führen wird, bleibt abzuwarten (Kanerva et al. 2012).

\section{Literatur}

Ammon A, Gastmeier P, Weist K, Kramer MH, Petersen LR (2001) Empfehlungen zur Untersuchung von Ausbrüchen nosokomialer Infektionen. Robert Koch-Institut, Heft 21

Askarian M, Mahmoudi H, Assadian O (2013) Incidence of nosocomial infections in a big university affiliated hospital in Shiraz, Iran: a six-month experience. Int J Prev Med 4:366-372

Astagneau P, Rioux C, Golliot F, Brucker G (2001) Morbidity and mortality associated with surgical site infections: results from the 1997-1999 INCISO surveillance. J Hosp Infect 48:267-274

Balasubramanian P, Tullu MS (2014) Study of ventilator-associated pneumonia in a pediatric intensive care unit. Indian $\mathrm{J}$ Pediatr $81: 1182-1186$
Barnett AG, Page K, Campell M et al (2013) The increased risks of death and extra length of hospital and ICU stay from hospitalacquired bloodstream infections: a case-control study. BMJ Open 3:e003587

Beck-Bornholdt HP, Dubben HH (2001) Der Hund, der Eier legt. Rowohlt, Taschenbuch Verlag

Behnia M, Logan SC, Fallen L, Catalano P (2014) Nosocomial and ventilator-associated pneumonia in a community hospital intensive care unit: a retrospective review and analysis. BMC Res Notes 7:232

Behnke M, Hansen S, Leistner R, Diaz LA, Gropmann A, Sohr D, Gastmeier P, Piening B (2013) Nosocomial infection and antibiotic use: a second national prevalence study in Germany. Dtsch Arztebl Int 110:627-633

Bekaert M, Timsit JF, Vansteelandt S, Depuydt P, Vesin A, GarrousteOrgeas $\mathrm{M}$ et al (2011) Attributable mortality of ventilator-associated pneumonia: a reappraisal using causal analysis. Am J Respir Crit Care Med 184:1133-1139

Beyersmann J, Gastmeier P, Grundmann H, Barwolff S, Geffers C, Behnke $\mathrm{M}$ et al (2006) Use of multistate models to assess prolongation of intensive care unit stay due to nosocomial infection. Infect Control Hosp Epidemiol 27:493-499

Blennow O, Ljungman P, Sparrelid E, Mattson J, Remberger M (2013) Incidence, risk factors, and outcome of bloodstream infections during the pre-engraftment phase in 521 allogenic heatopoietic stem cell transplantations. Transpl Infect Dis 16:106-114

Bueno-Cavanillas A, Delgado-Rodriguez M, Lopez-Luque A, Schaffino-Cano S, Galvez-Vargas R (1994) Influence of nosocomial infection on mortality rate in an intensive care unit. Crit Care Med 22:55-60

Chung M, Dickinson G, De Lencastre H, Tomasz A (2004) International clones of methicillin-resistant Staphylococcus aureus in two hospitals in Miami, Florida. J Clin Microbiol 42:542-547

Coughlin SS (1990) Recall bias in epidemiologic studies. J Clin Epidemiol 43:87-91

Daschner F, Nadjem H, Langmaack H, Sandritter W (1978) Surveillance, prevention and control of hospital-acquired infections. III. Nosocomial infections as cause of death: retrospective analysis of 1000 autopsy reports. Infection 6:261-265

Dettenkofer M, Seegers S, Antes G, Motschal EL, Schumacher M, Daschner F (2004) Does the architecture of hospital facilities influence nosocomial infection rates? A systematic review. Infect Control Hosp Epidemiol 25:21-25

Edwards JR, Peterson KD, Andrus ML, Dudeck MA, Pollock DA, Horan TC et al (2008) National Healthcare Safety Network (NHSN) Report, data summary for 2006 through 2007, issued November 2008. Am J Infect Control 36:609-626

European Centre for Disease Prevention and Control (ECDC) (2013) Point prevalence survey of healthcare-associated infections and antimicrobial use in European acute care hospitals 2011-2012. Issued 2013 by ECDC, coordinated by Carl Suetens

Fagon JY, Chastre J, Hance AJ, Montravers P, Novara A, Gibert C (1993) Nosocomial pneumonia in ventilated patients: a cohort study evaluating attributable mortality and hospital stay. Am J Med 94:281-288

Feinstein AR (1985) The architecture of clinical research. Saunders, Philadelphia, S 225-229

Garnacho J, Sole-Violan J, Sa-Borges M, Diaz E, Rello J (2003) Clinical impact of pneumonia caused by Acinetobacter baumannii in intubated patients: a matched cohort study. Crit Care Med 31:2478-2482

Gastmeier P, Kramer M (2004) Epidemiologie der Infektionen. In: Adam D, Doerr HW, Link H, Lode H (Hrsg) Die Infektiologie. Springer, Berlin/Heidelberg/New York/Tokio

Gastmeier P, Rüden H (2001) Epidemiologie und Surveillance nosokomialer Infektionen. In: Kramer A, Heeg P, Botzenhart K (Hrsg) Krankenhaus- und Praxishygiene. Urban \& Fischer, München 
Gastmeier P, Behnke M, Breier AC, Piening B, Schwab F, Dettenkofer M, Geffers C (2012) Healthcare-associated infection rates: measuring and comparing. Experiences from the German National Nosocomial Infection Surveillance System (KISS) and from other surveillance systems. Bundesgesundheitsblatt Gesundheitsforschung Gesundheitsschutz 55:1363-1369

Geffers C, Gastmeier P, Rüden H (2002) Nosokomiale Infektionen, Robert Koch-Institut, Gesundheitsberichterstattung des Bundes, 8

Geffers C, Sohr D, Gastmeier P (2008) Mortality attributable to hospitalacquired infections among surgical patients. Infect Control Hosp Epidemiol 29:1167-1170

Gordis L (2001) Epidemiologie. Verlag im Kilian, Marburg

Gross PA, Van Antwerpen C (1983) Nosocomial infections and hospital deaths. A case-control study. Am J Med 75:658-662

Grundmann H, Baerwolf S, Schwab F et al (2005) How many infections are caused by transmission in intensive care units? Crit Care Med 33:946-951

Guzman-Herrador B, Diaz Molina C, Allam MF, Fernandez-Crehuet Navajas R (2014) Underlying illness severity and outcome of nosocomial pneumonia: prospective cohort study in intensive care unit. $\mathrm{J}$ Hosp Infect 86:53-56

Haley RW, Culver DH, White JW et al (1985a) The efficacy of infection surveillance and control programs in preventing nosocomial infections in US hospitals. Am J Epidemiol 121:182-205

Haley RW, Tenney JH, Lindsey JO, Garner JS, Bennett JV (1985b) How frequent are outbreaks of nosocomial infection in community hospitals? Infect Control 6:233-236

Harbarth S, Sax H, Gastmeier P (2003) The preventable proportion of nosocomial infections: an overview of published reports. J Hosp Infect 54:258-266

Huoi C, Vanhems P, Nicolle MC, Michallet M, Benet T (2013) Incidence of hospital-acquired pneumonia, bacteraemia and urinary tract infections in patients with haematological malignancies, 2004-2010: a surveillance-based study. PLoS One 8:e58121

Jenks PJ, Laurent M, McQuarry S, Watkins R (2014) Clinical and economic burden of surgical site infection (SSI) and predicted financial consequences of elimination of SSI from an English hospital. J Hosp Infect 86:24-33

Kanerva M, Ollgren J, Hakanen AJ, Lyytikainen O (2012) Estimating the burden of healthcare-associated infections caused by selected multidrug-resistant bacteria Finland, 2010. Antimicrob Resist Infect Control 1:33

Kaye KS, Anderson DJ, Sloane R et al (2009) The impact of surgical site infection on older operative patients. J Am Geriatr Soc $57: 46-54$

Kaye KS, Marchaim D, Chen TY et al (2014) Effect of nosocomial bloodstream infections on mortality, length of stay, and hospital costs in older adults. J Am Geriatr Soc 62:306-311

Kiroff GK (2001) Publication bias in presentations to the Annual Scientific Congress. ANZ J Surg 71:167-171

Klompas M, Kleinman K, Murphy MV (2014) Descriptive epidemiology and attributable morbidity of ventilator-associated events. Infect Control Hosp Epidemiol 35:502-510

Kübler A, Duszynska W, Rosenthal VD, Fleischer M, Kaiser T, Szewczyk E, Barteczko-Grajek B (2012) Device-associated infection rates and extra length of stay in an intensive care unit of a university hospital in Wroclw, Poland: International Nosocomial Infection Control Consortium's (INICC) findings. J Crit Care 27:105.e5-105.e10

Laupland KB, Zygun DA, Davies HD et al (2002) Incidence and risk factors for acquiring nosocomial urinary tract infection in the critically ill. J Crit Care 17:50-57

Lee J, Singletary R, Schmader K, Anderson DJ, Bolognesi M, Kaye KS (2006) Surgical site infection in the elderly following orthopaedic surgery. J Bone Joint Surg 88:1705-1712
Lewis SM (1995) The effect of surveillance definitions on nosocomial urinary tract infection rates in a rehabilitation hospital. Infect Control Hosp Epidemiol 16:43-48

Liu CY, Liao CH, Chen YC, Chang SC (2010) Changing epidemiology of nosocomial bloodstream infections in 11 teaching hospitals in Taiwan between 1993 and 2006. J Microbiol Immunol Infect 43:416-429

Martinez-Resendez MF, Garza-Gonzalez E, Mendoza-Olazaran S, Herrera-Guerra A, Rodriguez-Lopez JM, Perez-Rodriguez E et al (2014) Impact of daily chlorhexidine baths and hand hygiene compliance on nosocomial infection rates in critically ill patients. Am J Infect Control 42:713-717

McGarry SA, Engemann JJ, Schmader K, Sexton DJ, Kaye KS (2004) Surgical-site infection due to staphylococcus aures among elderly patients: mortality, duration of hospitalization, and cost. Infect Control Hosp Epidemiol 25:461-467

Melsen WG, Rovers MM, Bonten MJ (2009) Ventilator-associated pneumonia and mortality: a systematic review of observational studies. Crit Care Med 37:2709-2718

Melsen WG, Rovers MM, Groenwold RH, Bergmans DC, Camus C, Bauer TT et al (2013) Attributable mortality of ventilator-associated pneumonia: a meta-analysis of individual patient data from randomised prevention studies. Lancet Infect Dis 13:665-671

Mitt P, Metswaht T, Adamson V, Telling K, Naaber P, Lutsar I, Maimets M (2014) Five-year prospective surveillance of nosocomial bloodstream infections in an Estonian paediatric intensive care unit. J Hosp Infect 86:95-99

Moro ML, Romi R, Severini C et al (2002) Patient-to-patient transmission of nosocomial malaria in Italy. Infect Control Hosp Epidemiol 23:338-341

Olaechea PM, Palomar M, Alvarez-Lerma F, Otal JJ, Insausti J, LopezPueyo MJ (2013) Morbidity and mortality associated with primary and catheter-related bloodstream infections in critically ill patients. Rev Esp Quimioter 26:21-29

Petrosillo N, Raffaele B, Martini L et al (2002) A nosocomial and occupational cluster of hepatitis A virus infection in a pediatric ward. Infect Control Hosp Epidemiol 23:343-345

Rello J, Ollendorf DA, Oster G, Vera-Llonch M, Bellm L, Redman R, Kollef MH (2002) Epidemiology and outcomes of ventilatorassociated pneumonia in a large US database. Chest 122:2115-2121

Rello J, Lorente C, Diaz E, Bodi M, Boque C, Sandiumenge A, Santamaria JM (2003) Incidence, etiology, and outcome of nosocomial pneumonia in ICU patients requiring percutaneous tracheotomy for mechanical ventilation. Chest 124:2239-2243

Roberts FJ, Walsh A, Wing P, Dvorak M, Schweigel J (1998) The influence of surveillance methods on surgical wound infection rates in a tertiary care spinal surgery service. Spine 23:366-370

Rogers AD, Deal C, Argent AC, Hudson DA, Rode H (2014) Ventilator associated pneumonia in major paediatric burns. Burns 40:1141-1148

Rosenthal VD, Guzman S, Orellano PW (2003) Nosocomial infections in medical-surgical intensive care units in Argentina: attributable mortality and length of stay. Am J Infect Control 31:291-295

Rüden H, Daschner F, Gastmeier P, Mielke M (2003) Definition nosokomialer Infektionen (CDC-Definitionen), Nationales Referenzzentrum für Surveillance von nosokomialen Infektionen. Robert KochInstitut, Berlin

Sopena N, Heras E, Casas I, Bechini J, Guasch I, Pedro-Botet ML et al (2014) Risk factors for hospital-acquired pneumonia outside the intensive care unit: a case-control study. Am J Infect Control 42:38-42

Stevens V, Geiger K, Concannon C, Nelson RE, Brown J, Dumyati G (2014) Inpatient costs, mortality and 30-day re-admission in patients with central-line-associated bloodstream infections. Clin Microbiol Infect 20:O318-O324

Timsit JF, Chevret S, Valcke J et al (1996) Mortality of nosocomial pneumonia in ventilated patients: influence of diagnostic tools. Am J Respir Crit Care Med 154:116-123 
Titsworth WL, Hester J, Correia T, Reed R, Williams M, Guin P et a (2012) Reduction of catheter-associated urinary tract infections among patients in a neurological intensive care unit: a single institution's success. J Neurosurg 116:911-920

Varia M, Wilson S, Sarwal S, McGeer A, Gournis E, Galanis E, Henry B (2003) Investigation of a nosocomial outbreak of severe acute respiratory syndrome (SARS) in Toronto, Canada. CMAJ 169:285-292

Verstraete E, Boelens J, De Coen K et al (2014) Healthcare-associated bloodstream infections in a neonatal intenisve care unit over a 20-year period (1992-2011): trends in incidence, pathogens, and mortality. Infect Control Hosp Epidemiol 35:511-518
Vineis P (1999) Ras mutations and a cup of coffee: cause, confounder, effect modifier, or what else? J Epidemiol Community Health 53:685

Wenzel RP, Nettleman MD (1999) Principles of hospital epidemiology. In: Mayhall CG (Hrsg) Hospital epidemiology and infection control. Lippincott Williams \& Wilkins, Philadelphia

Wenzel RP, Thompson RL, Landry SM, Russell BS, Miller PJ, Ponce DL, Miller GB Jr (1983) Hospital-acquired infections in intensive care unit patients: an overview with emphasis on epidemics. Infect Control 4:371-375

Zimlichman E, Henderson D, Tamir O et al (2013) Heath careassociated infections - a meta-analysis of costs and financial impact on the US health care system. JAMA Intern Med 173:2039-2046 